\title{
Inverse- $C$-class function on weak semi compatibility and fixed point theorems for expansive mappings in $G$-metric spaces
}

\author{
Arslan Hojat Ansari, Mukesh Kumar Jain, Naeem Saleem
}

\begin{abstract}
In this paper we introduce the concept of inverse $C$-class function in $G$-metric setting and established some fixed point theorems. We also put some examples in support of proved fixed point results.
\end{abstract}

\section{INTRODUCTION AND MATHEMATICAL PRELIMINARIES}

The study of metric fixed point have played very vital role with the applications in mathematics and applied science. Later it was found very essential to generalize the notion named metric space by many researchers. With this respect many generalization have come in the metric space like, $D$-metric space, 2-metric space, cone metric space, fuzzy metric space and manger space, etc. The 2-metric space was proposed by Gahler [12] and considered an independent theory rather than a generalization of metric space. One more generalization came in exist called $D$-metric space. It was introduced by Dhage [11]. He published lot of papers concerned to generalization of metric space, but many of his results found to be considered erroneous. This fact was set on by Mustafa and then, in collaboration with Sims [15] they demonstrated an improved form called $G$-metric space. Later Mustafa did more work in collaboration with Obiedat, Awahdeh, Shatanawi and Karpinar. For more information one can go through [13]-[21].

In the stream of generalization, in 2014 the concept of $C$-class function was introduced by Ansari [3]. Using this concept many fixed point results were generalized in different spaces. For more information one can go through [4][10]. This concept has enticed many fixed point theorists. Later the more generalized form called inverse $C$-class function was introduced by Saleem et al. [24].

The purpose of the present paper is to extend the concept of inverse $C$ class function in $G$-metric setting and proved some fixed point theorems by

2010 Mathematics Subject Classification. Primary 47H10; Secondary 54H25.

$K e y$ words and phrases. Common fixed point, inverse- $C$-class function.

Full paper. Received 5 June 2019, revised 15 October 2019, accepted 9 April 2020, available online 4 May 2020. 
taking weak semi compatibility in $G$-metric space. These results include compatible mappings of type (E) also different compatible and commuting mappings.

Definition 1 ([13]). Let $X$ be a nonempty set and $G: X \times X \times X \rightarrow \mathbb{R}^{+}$ be a function satisfying the following properties:

(G1) $G(x, y, z)=0$ iff $x=y=z$;

(G2) $0<G(x, x, y)$, for all $x, y \in X$ with $x \neq y$;

(G3) $G(x, x, y) \leq G(x, y, z)$, for all $x, y, z \in X$ with $z \neq y$;

(G4) $G(x, y, z)=G(p\{x, y, z\})$, where $p$ is any permutation of $x, y, z$ (symmetry in all three variables);

(G5) $G(x, y, z) \leq G(x, a, a)+G(a, y, z)$, for all $x, y, z, a \in X$ (rectangle inequality).

Then, the function $G$ is called a $G$-metric on $X$ and the pair $(X, G)$ is called a $G$-metric space.

In 2014, the concept of $C$-class functions was introduced by A. H. Ansari [3]. By using this concept, we can generalize many fixed point theorems in the literature.

Definition 2 ([25]). Mappings $f$ and $g$ of a metric space $(X, d)$ is said to be weak semi compatible if $\lim _{n \rightarrow \infty} f g x_{n}=g t$ or $\lim _{n \rightarrow \infty} g f x_{n}=f t$, whenever a sequence $\left\{x_{n}\right\}$ in $X$ such that $\lim _{n \rightarrow \infty} f x_{n}=\lim _{n \rightarrow \infty} g x_{n}=t$, for some $t$ in $X$.

Definition 3 ([23]). A self map pair $(f, g)$ of a metric space $(X, d)$ is said to be $R$-weak commuting of type $\left(A_{f}\right)$ if there exists some positive real number $R$ such that $d(f g x, g g x) \leq R d(f x, g x)$, for all $x \in X$. Similarly, a self map pair $(f, g)$ of a metric space $(X, d)$ is said to be $R$-weak commuting of type $\left(A_{g}\right)$ if there exists some positive real number $R$ such that $d(g f x, f f x) \leq$ $R d(f x, g x)$, for all $x \in X$.

Definition 4 ([26]). Self mappings $f$ and $g$ are called $f$-compatible of type (E) if $\lim _{n \rightarrow \infty} f f x_{n}=\lim _{n \rightarrow \infty} f g x_{n}=g t$, whenever a sequence $\left\{x_{n}\right\}$ in $X$ such that $\lim _{n \rightarrow \infty} f x_{n}=\lim _{n \rightarrow \infty} g x_{n}=t$, for some $t$ in $X$. Similarly, self mappings $f$ and $g$ are called $g$-compatible of type (E) if $\lim _{n \rightarrow \infty} g g x_{n}=\lim _{n \rightarrow \infty} g f x_{n}=g t$, whenever a sequence $\left\{x_{n}\right\}$ in $X$ such that $\lim _{n \rightarrow \infty} f x_{n}=\lim _{n \rightarrow \infty} g x_{n}=t$, for some $t$ in $X$.

Definition 5 ([22]). Two self mappings $f$ and $g$ are called $f$-compatible if $\lim _{n \rightarrow \infty} d\left(f g x_{n}, g g x_{n}\right)=0$, whenever a sequence $\left\{x_{n}\right\}$ in $X$ such that $\lim _{n \rightarrow \infty} f x_{n}=$ $\lim _{n \rightarrow \infty} g x_{n}=t$, for some $t$ in $X$. Similarly, self mappings $f$ and $g$ are called $f$-compatible if $\lim _{n \rightarrow \infty} d\left(f g x_{n}, g g x_{n}\right)=0$, whenever a sequence $\left\{x_{n}\right\}$ in $X$

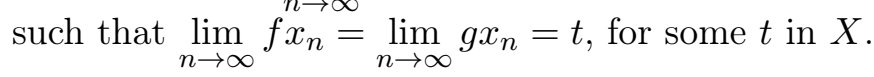


Definition 6 ([1]). Mappings $f$ and $g$ satisfy E.A. property if there exists a sequence $\left\{x_{n}\right\} \in X$ which satisfy $\lim _{n \rightarrow \infty} f x_{n}=\lim _{n \rightarrow \infty} g x_{n}=t$, for some $t$ in $X$.

Definition $7([3])$. A mapping $f:[0, \infty)^{2} \rightarrow \mathbb{R}$ is called $C$-class function if it is continuous and satisfies following axioms:

(1) $f(s, t) \leq s$

(2) $f(s, t)=s$ implies that either $s=0$ or $t=0$, for all $s, t \in[0, \infty)$.

Note for some $F$ we have that $F(0,0)=0$.

We denote $C$-class functions as $\mathcal{C}$.

Definition 8 ([24]). A mapping $F:[0, \infty)^{2} \rightarrow \mathbb{R}$ is called inverse- $C$-class function if it is continuous and satisfies following axioms:

(1) $F(s, t) \geq s$;

(2) $F(s, t)=s$ implies that either $s=0$ or $t=0$, for all $s, t \in[0, \infty)$.

Note for some $F$ we have that $F(0,0)=0$.

We denote inverse $C$-class functions as $\mathcal{C}_{\text {inv }}$.

Let $\Phi$ denote the set of all functions $\varphi:[0,+\infty) \rightarrow[0,+\infty)$ that satisfy the following conditions:

(1) $\varphi$ is lower semi-continuous on $[0,+\infty)$;

(2) $\varphi(0)=0$;

(3) $\varphi(s)>0$ for each $s>0$.

Let $\Phi_{1}$ denote the set of all functions $\varphi:[0,+\infty) \rightarrow[0,+\infty)$ that satisfy the following conditions:

(1) $\varphi$ is lower semi-continuous on $[0,+\infty)$;

(2) $\varphi(0) \geq 0$

(3) $\varphi(s)>0$ for each $s>0$.

Let $\Psi$ denote all the functions $\psi:[0, \infty) \rightarrow[0, \infty)$ which satisfy:

(i) $\psi(t)=0$ if and only if $t=0$;

(ii) $\psi$ is continuous and monotinic increasing.

\section{Main Results}

Theorem 1. Let $f$ and $g$ are weak semi compatible, $R$-weak commuting of type $A_{f}$ self mappings of complete $G$-metric space $(X, G)$ and suppose that $f: \cup_{i=1}^{m} A_{i} \rightarrow \cup_{i=1}^{m} A_{i}$ satisfies the following conditions: $\left(\right.$ where $\left.A_{m+1}=A_{1}\right)$ :

(a) $f(X) \subseteq g(X)$;

(b) For all $x, y, z \in X$ holds

$$
\psi(G(g x, g y, g z)) \geq F(\psi(G(f x, f y, f z)), \varphi(G(f x, f y, f z))) ;
$$

(c) $f$ and $g$ are either $f$-compatible of type $(E)$ or $g$-compatible of type $(E)$. 
If functions $\varphi \in \Phi, \psi \in \Psi$ and $F \in \mathcal{C}_{\text {inv }}$ then $f$ and $g$ have a common fixed point in $X$.

Proof. Let $x_{0}$ be any point in $X$. Since $f(X) \subseteq g(X)$, there exist a point $x_{1}$ in $X$ such that $f x_{0}=g x_{1}$. Similarly, we can find a sequence $f x_{n}$ in $X$ such that $f x_{n}=g x_{n+1}$. Let $z_{n}=f x_{n}=g x_{n+1}$. Now we have to show that $\left\{z_{n}\right\}$ is a Cauchy sequence in $X$.

Step 1. We will prove that $\lim _{n \rightarrow \infty} G\left(z_{n}, z_{n+1}, z_{n+1}\right)=0$.

For proving this, we take condition (b) with $x=x_{n}, y=x_{n+1}$ and $z=$ $x_{n+1}$, so we have

$$
\begin{aligned}
& \psi\left(G\left(z_{n-1}, z_{n}, z_{n}\right)\right)=\psi\left(G\left(g x_{n}, g x_{n+1}, g x_{n+1}\right)\right) \geq \\
& \geq F\left(\psi\left(G\left(f x_{n}, f x_{n+1}, f x_{n+1}\right)\right), \varphi\left(G\left(f x_{n}, f x_{n+1}, f x_{n+1}\right)\right)\right) \\
& =F\left(\psi\left(G\left(z_{n}, z_{n+1}, z_{n+1}\right)\right), \varphi\left(G\left(z_{n}, z_{n+1}, z_{n+1}\right)\right)\right) \\
& \geq \psi\left(G\left(z_{n}, z_{n+1}, z_{n+1}\right)\right)
\end{aligned}
$$

and therefore

$$
G\left(z_{n}, z_{n+1}, z_{n+1}\right) \leq G\left(z_{n-1}, z_{n}, z_{n}\right) .
$$

Hence, we conclude that $\left\{G\left(z_{n}, z_{n+1}, z_{n+1}\right)\right\}$ is a nondecreasing sequence of non-negative real numbers. Thus, there exist a $r \geq 0$ such that

$$
\lim _{n \rightarrow \infty} G\left(z_{n}, z_{n+1}, z_{n+1}\right)=r .
$$

Letting $n \rightarrow \infty$ in (2) and by the continuity of $\psi$ and $\varphi$, we have

$$
\psi(r) \geq F(\psi(r), \varphi(r)) \geq \psi(r)
$$

which implies either $\psi(r)=0$ or $\varphi(r)=0$. This gives

$$
\lim _{n \rightarrow \infty} G\left(z_{n}, z_{n+1}, z_{n+1}\right)=0 .
$$

Step 2. We will show that $\left\{z_{n}\right\}$ is a $G$-Cauchy sequence in $X$. Therefore, we will show that for every $\varepsilon>0$, there exists $k \in \mathbb{N}$ such that for all $m, n \geq k$, $G\left(z_{m}, z_{n}, z_{n}\right)<\varepsilon$.

Suppose that the above statement is false. Then, there exists $\varepsilon>0$ for which we can find subsequences $\left\{z_{m(k)}\right\}$ and $\left\{z_{n(k)}\right\}$ of $\left\{z_{n}\right\}$ such that $n(k)>m(k) \geq k$ :

(a) $m(k)=3 t$ and $n(k)=3 t^{\prime}+1$, where $t$ and $t^{\prime}$ are nonnegative integers such that

$$
G\left(z_{m(k)}, z_{n(k)}, z_{n(k)}\right) \geq \varepsilon .
$$

(b) $n(k)$ is the smallest number such that the condition (b) holds:

$$
G\left(z_{m(k)}, z_{n(k)-1}, z_{n(k)-1}\right)<\varepsilon .
$$


From rectangle inequality and (7), we have

$$
\begin{aligned}
& G\left(z_{m(k)}, z_{n(k)}, z_{n(k)}\right) \leq \\
& \leq G\left(z_{m(k)}, z_{n(k)-1}, z_{n(k)-1}\right)+G\left(z_{n(k)-1}, z_{n(k)}, z_{n(k)}\right)< \\
& <\varepsilon+G\left(z_{n(k)-1}, z_{n(k)}, z_{n(k)+1}\right) .
\end{aligned}
$$

If $k \rightarrow \infty$ in (8), from (5) and (7) we conclude that

$$
\lim _{k \rightarrow \infty} G\left(z_{m(k)}, z_{n(k)}, z_{n(k)}\right)=\varepsilon .
$$

Again, from rectangle inequality

$$
\begin{aligned}
& G\left(z_{m(k)}, z_{n(k)}, z_{n(k)+1}\right) \leq \\
& \leq G\left(z_{m(k)}, z_{n(k)}, z_{n(k)}\right)+G\left(z_{n(k)}, z_{n(k)}, z_{n(k)+1}\right) \leq \\
& \leq G\left(z_{m(k)}, z_{n(k)}, z_{n(k)}\right)+G\left(z_{n(k)}, z_{n(k)+1}, z_{n(k)+2}\right)
\end{aligned}
$$

and

$$
G\left(z_{m(k)}, z_{n(k)}, z_{n(k)}\right) \leq G\left(z_{m(k)}, z_{n(k)}, z_{n(k)+1}\right) .
$$

Hence in (10) and (11), if $k \rightarrow \infty$, using (5), (6) and (9), we have

$$
\lim _{k \rightarrow \infty} G\left(z_{m(k)}, z_{n(k)}, z_{n(k)+1}\right)=\varepsilon .
$$

On the other hand

$$
\begin{aligned}
& G\left(z_{m(k)}, z_{n(k)+1}, z_{n(k)+1}\right) \leq \\
& \leq G\left(z_{m(k)}, z_{n(k)}, z_{n(k)}\right)+G\left(z_{n(k)}, z_{n(k)+1}, z_{n(k)+1}\right)
\end{aligned}
$$

and

$$
\begin{aligned}
& G\left(z_{n(k)}, z_{n(k)+1}, z_{m(k)}\right) \leq \\
& \leq G\left(z_{n(k)}, z_{n(k)+1}, z_{n(k)+1}\right)+G\left(z_{n(k)+1}, z_{n(k)+1}, z_{m(k)}\right) .
\end{aligned}
$$

Hence from (13) and (14), by applying limit $k \rightarrow \infty$, from (5), (9) and (12), we have

$$
\lim _{k \rightarrow \infty} G\left(z_{m(k)}, z_{n(k)+1}, z_{n(k)+1}\right)=\varepsilon .
$$

In a similar way, we have

$$
\begin{aligned}
& G\left(z_{m(k)+1}, z_{n(k)}, z_{n(k)+1}\right) \leq \\
& \leq G\left(z_{m(k)+1}, z_{m(k)}, z_{m(k)}\right)+G\left(z_{m(k)}, z_{n(k)}, z_{n(k)+1}\right) \leq \\
& \leq 2 G\left(z_{m(k)}, z_{m(k)+1}, z_{m(k)+1}\right)+G\left(z_{m(k)}, z_{n(k)}, z_{n(k)+1}\right)
\end{aligned}
$$

and

$$
\begin{aligned}
& G\left(z_{m(k)}, z_{n(k)}, z_{n(k)+1}\right) \leq \\
& \leq G\left(z_{m(k)}, z_{m(k)+1}, z_{m(k)+1}\right)+G\left(z_{m(k)+1}, z_{n(k)}, z_{n(k)+1}\right),
\end{aligned}
$$

from (16) and (17) by taking limit as $k \rightarrow \infty$ and using (5) and (12), we have

$$
\lim _{k \rightarrow \infty} G\left(z_{m(k)+1}, z_{n(k)}, z_{n(k)+1}\right)=\varepsilon .
$$


Also

$$
G\left(z_{m(k)}, z_{n(k)+1}, z_{n(k)+1}\right) \leq G\left(z_{m(k)}, z_{m(k)+1}, z_{n(k)+1}\right)
$$

and

$$
\begin{aligned}
& G\left(z_{m(k)}, z_{m(k)+1}, z_{n(k)+1}\right) \leq \\
& \leq G\left(z_{m(k)}, z_{m(k)+1}, z_{m(k)+1}\right)+G\left(z_{m(k)+1}, z_{m(k)+1}, z_{n(k)+1}\right) \leq \\
& \leq G\left(z_{m(k)}, z_{m(k)+1}, z_{m(k)+1}\right)+G\left(z_{m(k)+1}, z_{n(k)}, z_{n(k)+1}\right) .
\end{aligned}
$$

If $k \rightarrow \infty$ in (19) and (20), also using (5), (15) and (17), we have

$$
\lim _{k \rightarrow \infty} G\left(z_{m(k)}, z_{m(k)+1}, z_{n(k)+1}\right)=\varepsilon .
$$

Further

$$
G\left(z_{m(k)+1}, z_{n(k)+1}, z_{n(k)+1}\right) \leq G\left(z_{m(k)+1}, z_{n(k)+1}, z_{n(k)}\right)
$$

and

$$
\begin{aligned}
& G\left(z_{m(k)+1}, z_{n(k)}, z_{n(k)+1}\right) \leq \\
& \leq G\left(z_{m(k)+1}, z_{n(k)+1}, z_{n(k)+1}\right)+G\left(z_{n(k)+1}, z_{n(k)+1}, z_{n(k)}\right) .
\end{aligned}
$$

So, from (5), (18), (21) and (22), we have

$$
\lim _{k \rightarrow \infty} G\left(z_{m(k)+1}, z_{n(k)+1}, z_{n(k)+1}\right)=\varepsilon .
$$

Finally

$$
\begin{aligned}
& G\left(z_{m(k)+1}, z_{n(k)+1}, z_{n(k)+2}\right) \leq \\
& \leq G\left(z_{m(k)+1}, z_{n(k)+1}, z_{n(k)+1}\right)+G\left(z_{n(k)+1}, z_{n(k)+1}, z_{n(k)+2}\right) \leq \\
& \leq G\left(z_{m(k)+1}, z_{n(k)+1}, z_{n(k)+1}\right)+G\left(z_{n(k)}, z_{n(k)+1}, z_{n(k)+2}\right)
\end{aligned}
$$

and

$$
G\left(z_{m(k)+1}, z_{n(k)+1}, z_{n(k)+1}\right) \leq G\left(z_{m(k)+1}, z_{n(k)+1}, z_{n(k)+2}\right) .
$$

Hence, in (25) and (26), if $k \rightarrow \infty$ and using (5) and (24), we have

$$
\lim _{k \rightarrow \infty} G\left(y_{m(k)+1}, z_{n(k)+1}, z_{n(k)+2}\right)=\varepsilon .
$$

Now, suppose $x=x_{m(k)+1}, y=x_{n(k)+1}$ and $z=x_{n(k)+1}$ in (1), for all $k \geq 0$, we have

$$
\begin{aligned}
& \psi\left[G\left(z_{m(k)}, z_{n(k)}, z_{n(k)}\right)\right]=\psi\left[G\left(g x_{m(k)+1}, g x_{n(k)+1}, g x_{n(k)+1}\right)\right] \geq \\
& \left.\geq F\left[\psi\left(G\left(f x_{m(k)+1}, f x_{n(k)+1}, f x_{n(k)+1}\right)\right), \varphi G\left(f x_{m(k)+1}, f x_{n(k)+1}, f x_{n(k)+1}\right)\right)\right] \\
& =F\left[\psi\left(G\left(z_{m(k)+1}, z_{n(k)+1}, z_{n(k)+1}\right)\right), \varphi\left(G\left(z_{m(k)+1}, z_{n(k)+1}, z_{n(k)+1}\right)\right)\right] .
\end{aligned}
$$

Now, from (9) and (24), if $k \rightarrow \infty$ in (28), we have

$$
\psi(\varepsilon) \geq F(\psi(\varepsilon), \varphi(\varepsilon)) \geq \psi(\varepsilon),
$$

so $\psi(\varepsilon)=0$ or $\varphi(\varepsilon)=0$. Hence, $\varepsilon=0$ is a contradiction. Consequently, $\left\{z_{n}\right\}$ is a $G$-Cauchy sequence. 
Since $(X, G)$ is complete, there exists a point $t \in X$ such that $\lim _{n \rightarrow \infty} f x_{n}=$ $\lim _{n \rightarrow \infty} g x_{n+1}=t$.

Case 1. If $f$ and $g$ are $f$-compatible of type (E).

Since $f$ and $g$ are weak semi compatible mappings, this yields $\lim _{n \rightarrow \infty} f g x_{n}=$ $g t$ or $\lim _{n \rightarrow \infty} g f x_{n}=f t$. First, we take $\lim _{n \rightarrow \infty} g f x_{n}=f t$. Since $f$ and $g$ are $f$-compatible of type $(E)$ then $\lim _{n \rightarrow \infty} f f x_{n}=\lim _{n \rightarrow \infty} f g x_{n}=g t$, Now, by (b),

$$
\psi\left[G\left(g f x_{n}, g t, g t\right)\right] \geq F\left[\psi\left(G\left(f f x_{n}, f t, f t\right)\right), \varphi\left(G\left(f f x_{n}, f t, f t\right)\right)\right] .
$$

On limiting $n \rightarrow \infty$, we have

$$
\psi[G(f t, g t, g t)] \geq F[\psi(G(g t, f t, f t)), \varphi(G(g t, f t, f t))] .
$$

Since $(X, G)$ is symmetric $G$-metric space, this yields

$$
\psi[G(f t, g t, g t)] \geq F[\psi(G(f t, g t, g t)), \varphi(G(f t, g t, g t))] .
$$

Then by definition of function we have,

$$
\psi[G(f t, g t, g t)] \geq F[\psi(G(f t, g t, g t)), \varphi(G(f t, g t, g t))] \geq \psi[G(f t, g t, g t)] .
$$

This implies further $\psi(G(f t, g t, g t))=0$ or $\varphi(G(f t, g t, g t))=0$. This yields $G(f t, g t, g t)=0$ or $f t=g t$. $R$-weak commutativity of type $A_{f}$ yields

$$
d(f g t, g g t) \leq R d(f t, g t),
$$

which implies further $f g t=g f t$ or $f g t=g f t=f f t=g g t$. Then by (b) we have

$$
\begin{aligned}
\psi[G(g f t, g t, g t)] & \geq F[\psi(G(f f t, f t, f t)), \varphi(G(f f t, f t, f t))], \\
\psi[G(f f t, f t, f t)] & \geq F[\psi(G(f f t, f t, f t)), \varphi(G(f f t, f t, f t))] \\
& \geq \psi[G(f f t, f t, f t)] .
\end{aligned}
$$

This implies $\psi(G(f f t, f t, f t))=0$ or $\varphi(G(f f t, f t, f t))=0$ and gives finally $f f t=f t$. Therefore, $f f t=g f t=f t$ and $f t$ is common fixed point of $f$ and $g$.

Now, we take $\lim f g x_{n}=g t$. Since $f$ and $g$ are $f$-compatible of type (E), we have $\lim f f x_{n}=\lim f g x_{n}=g t$. Moreover, since $f$ and $g$ are $R$-weak commuting of type $A_{f}$, it yields $d\left(f g x_{n}, g g x_{n}\right) \leq R d\left(f x_{n}, g x_{n}\right)$. Now, limiting $n \rightarrow \infty$ we have $d\left(g t, \lim g g x_{n}\right) \leq R d(t, t)$, which implies $\lim g g x_{n}=g t$. Now, by (b)

$$
\psi\left[G\left(g g x_{n}, g t, g t\right)\right] \geq F\left[\psi\left(G\left(f g x_{n}, f t, f t\right)\right), \varphi\left(G\left(f g x_{n}, f t, f t\right)\right)\right] .
$$

Now limiting $n \rightarrow \infty$ yields

$$
\psi[G(g t, g t, g t)] \geq F[\psi(G(g t, f t, f t)), \varphi(G(g t, f t, f t))]
$$

or

$$
\psi(0) \geq F[\psi(G(g t, f t, f t)), \varphi(G(g t, f t, f t))] \geq \psi(G(g t, f t, f t)) .
$$


Since $\psi$ is monotonically increasing function, this yields

$$
0 \geq G(g t, f t, f t) \text { or } G(g t, f t, f t)=0
$$

and this implies $f t=g t$. By the preceding work it can be easily shown that $f t$ is common fixed point of $f$ and $g$.

Case 2. Let $f$ and $g$ be $g$-compatible of type $(E)$. Since $f$ and $g$ are weak semi compatible mappings then $\lim f g x_{n}=g t$ or $\lim g f x_{n}=f t$.

First, we take $\lim g f x_{n}=f t$. Since $f$ and $g$ are $g$-compatible of type (E), this yields $\lim g g x_{n}=\lim g f x_{n}=f t$. Moreover, since $f$ and $g$ are $R$-weak commuting of type $A_{f}$, this yields

$$
d\left(f g x_{n}, g g x_{n}\right) \leq R d\left(f x_{n}, g x_{n}\right) .
$$

On limiting $n \rightarrow \infty$, we get

$$
d\left(\lim f g x_{n}, f t\right) \leq R d(t, t)
$$

or $\lim f g x_{n}=f t$. Since $f(X) \subseteq g(X)$, there exists a point $u \in X$ such that $f t=g u$. Now by (b) we have

$$
\psi\left[G\left(g g x_{n}, g u, g u\right)\right] \geq F\left[\psi\left(G\left(f g x_{n}, f u, f u\right)\right), \varphi\left(G\left(f g x_{n}, f u, f u\right)\right)\right] .
$$

Limiting $n \rightarrow \infty$ yields

$$
\psi[G(f t, g u, g u)] \geq F[\psi(G(f t, f u, f u)), \varphi(G(f t, f u, f u))],
$$

which implies $\psi(G(g u, g u, g u)) \geq \psi(G(g u, f u, f u))$ or $\psi(0) \geq \psi(G(g u, f u, f u))$. Since $\psi$ is monotonically increasing, this yields $0 \geq G(g u, f u, f u)$ or $f u=$ $g u$. Now, it can be easily shown that $f u$ is common fixed point of $f$ and $g$.

Now, we take $\lim f g x_{n}=g t$. By (b), we have

$$
\psi\left[G\left(g g x_{n}, g t, g t\right)\right] \geq F\left[\psi\left(G\left(f g x_{n}, f t, f t\right)\right), \varphi\left(G\left(f g x_{n}, f t, f t\right)\right)\right] .
$$

Limiting $n \rightarrow \infty$ yields

$$
\psi[G(f t, g t, g t)] \geq F[\psi(G(g t, f t, f t)), \varphi(G(g t, f t, f t))] .
$$

Since $(X, G)$ is symmetric $G$-metric space, this yields

$$
\psi[G(g t, f t, f t)] \geq F[\psi(G(g t, f t, f t), \varphi(G(g t, f t, f t))] \geq \psi[G(g t, f t, f t)] .
$$

This implies further $\psi(G(g t, f t, f t))=0$ or $\varphi(G(g t, f t, f t))=0$, therefore $f t=g t$.

Now it can be easily shown that $f t$ is common fixed point of $f$ and $g$.

Example 1. Let $(X, G)$ be a $G$-metric space where $G$ defines as $G(x, y, z)=$ $|x-y|+|y-z|+|z-x|$, for all $x, y, z \in X$. Let $X=[1,5]$ and we define mapping $f, g: X \rightarrow X$ by

$$
f x=\left\{\begin{array}{cl}
1, & x=1 \text { or } x \in[3,5], \\
\frac{x+5}{2}, & x \in(1,3),
\end{array} \quad g x=\left\{\begin{array}{cl}
1, & x=1 \text { or } x=3, \\
x+2, & x \in(1,3), \\
x, & x \in(3,5] .
\end{array}\right.\right.
$$

Here functions $f$ and $g$ satisfy all the conditions of theorems with 1 is fixed point. To show weak semi compatibility and $f$-compatibility of type (E) 
of mappings $f$ and $g$, a sequence $1+\epsilon_{n}$ is taken where $\epsilon_{n} \rightarrow \infty$ whenever $n \rightarrow \infty$. Here $\lim _{n \rightarrow \infty} f x_{n}=\lim _{n \rightarrow \infty} g x_{n}=3$ then $\lim _{n \rightarrow \infty} f g x_{n}=1=g(3)$ and $\lim _{n \rightarrow \infty} g f x_{n}=3 \neq f(3)$ also $\lim _{n \rightarrow \infty} f f x_{n}=1=g(3)$. Moreover functions $f$ and $g$ satisfy $R$-weak commuting of type $A_{f}$.

Theorem 2. Let $f$ and $g$ be two weak semi compatible, $R$-weak commuting of type $A_{f}$ self mappings of $G$-metric space $(X, G)$ satisfying following conditions:

(a) $f(X) \subseteq g(X)$;

(b) For all $x, y, z \in X$;

$$
\psi(G(g x, g y, g z)) \geq F(\psi(G(f x, f y, f z), \varphi(G(f x, f y, f z))) ;
$$

(c) $f$ and $g$ are either $f$-compatible or $g$-compatible;

(d) $f$ and $g$ satisfy E.A. property.

If $\varphi \in \Phi, \psi \in \Psi$ and $F \in \mathcal{C}_{\text {inv }}$ then $f$ and $g$ have a common fixed point in $X$.

Proof. Since $f$ and $g$ satisfy E.A. property, then there exists a sequence $\left\{x_{n}\right\}$ in $X$ such that $\lim f x_{n}=\lim g x_{n}=t$ for some $t$ in $X$. Since $f(X) \subseteq g(X)$, then there exists a sequence $\left\{y_{n}\right\} \in X$ such that $\lim f x_{n}=\lim g y_{n}$ or $\lim f x_{n}=\lim g y_{n}=t$. Now by (b) we have

$$
\psi\left(G\left(g x_{n}, g y_{n}, g y_{n}\right)\right) \geq F\left(\psi\left(G\left(f x_{n}, f y_{n}, f y_{n}\right), \varphi\left(G\left(f x_{n}, f y_{n}, f y_{n}\right)\right)\right) .\right.
$$

Limiting $n \rightarrow \infty$ and by the definition of functions $F$ and $\psi$ yields $\lim f y_{n}=$ $t$. Thus

$$
\lim f x_{n}=t, \lim g x_{n}=t, \lim g y_{n}=t \text { and } \lim f y_{n}=t .
$$

Case 1. If $f$ and $g$ are $f$-compatible.

Since $f$ and $g$ are weak semi compatible mappings, this yields either $\lim f g x_{n}=g t$ or $\lim g f x_{n}=f t$. First, we take $\lim f g x_{n}=g t$. Since $f$ and $g$ are $f$-compatible, this yields $\lim f g x_{n}=\lim g g x_{n}$. It gives $\lim g g x_{n}=g t$. Now by (b),

$$
\psi\left(G\left(g g x_{n}, g t, g t\right)\right) \geq F\left(\psi\left(G\left(f g x_{n}, f t, f t\right), \varphi\left(G\left(f g x_{n}, f t, f t\right)\right)\right) .\right.
$$

Limiting $n \rightarrow \infty$ we get,

$$
\psi(G(g t, g t, g t)) \geq F(\psi(G(g t, f t, f t), \varphi(G(g t, f t, f t))) .
$$

By the definition of $F$ and $\psi$, it can be easily obtained $f t=g t$. Since $f$ and $g$ are $R$-weak commuting of type $A_{f}$, this gives

$$
d(f g t, g g t) \leq R d(f t, g t) .
$$

It further implies

$$
f g t=g f t \text { or } f g t=g f t=f f t=g g t .
$$

Now by (b), it is very easy to get $f f t=g f t=f t$ or $f t$ is common fixed point of $f$ and $g$. 
Next we take $\lim g f x_{n}=f t$. This yields by $30, \lim g g y_{n}=f t$. Since $f(X) \subseteq g(X)$. Then there exists a point $u$ in $X$ such that $f t=g u$. Now $R$ weak commuting of type $A_{f}$ yields $d\left(f g y_{n}, g g y_{n}\right) \leq R d\left(f y_{n}, g y_{n}\right)$. Limiting $n \rightarrow \infty$ yields $d\left(\lim f g y_{n}, g u\right) \leq R d(t, t)$. It further gives $\lim f g y_{n}=g u$. Then by (b)

$$
\psi\left(G\left(g g y_{n}, g u, g u\right)\right) \geq F\left(\psi\left(G\left(f g y_{n}, f u, f u\right), \varphi\left(G\left(f g y_{n}, f u, f u\right)\right)\right) .\right.
$$

Limiting $n \rightarrow \infty$ yields

$$
\psi(G(g u, g u, g u)) \geq F(\psi(G(g u, f u, f u), \varphi(G(g u, f u, f u))) .
$$

By the definition of $F$ and $\psi$, it can be easily obtained $f u=g u$. By (b) with $R$-weak commuting of type $A_{f}$, it can be easily shown that $f u$ is common fixed point of $f$ and $g$.

Case 2. if $f$ and $g$ are $g$-compatible.

Since $f$ and $g$ are weak semi compatible mappings, this yields either $\lim f g x_{n}=g t$ or $\lim g f x_{n}=f t$. First, we take $\lim f g x_{n}=g t$. By virtue of (30), $\lim f f x_{n}=g t$. Since $f$ and $g$ are $g$-compatible, this yields $\lim f g x_{n}=$ $\lim f f x_{n}$ or $\lim g f x_{n}=g t$. Then by (b)

$$
\psi\left(G\left(g f x_{n}, g t, g t\right)\right) \geq F\left(\psi\left(G\left(f f x_{n}, f t, f t\right), \varphi\left(G\left(f f x_{n}, f t, f t\right)\right)\right) .\right.
$$

Limiting $n \rightarrow \infty$ yields

$$
\psi(G(g t, g t, g t)) \geq F(\psi(G(g t, f t, f t), \varphi(G(g t, f t, f t))) .
$$

By the definition of $F$ and $\psi$, it can be easily shown that $f t=g t$. Then by (b) with $R$-weak commuting of type $A_{f}$, it can be easily shown that $f t$ is common fixed point of $f$ and $g$.

Finally we take $\lim g f x_{n}=f t$. Since $f(X) \subseteq g(X)$. Then there exists a point $u$ in $X$ such that $f t=g u$. By the previous working it is easy to show that $f u$ is common fixed point of $f$ and $g$.

Example 2. Let $(X, G)$ be a $G$-metric space where $G(x, y, z)$ be defined as $G(x, y, z)=|x-y|+|y-z|+|z-x|$, for all $x, y, z \in X$. Let $X=[1,4]$ and we define mapping $f, g: X \rightarrow X$ by

$$
f x=\left\{\begin{array}{ll}
1, & x=1, \\
2, & x \in(1,2], \\
3, & x \in(2,4],
\end{array} \quad g x=\left\{\begin{array}{cl}
1, & x=1, \\
x+1, & x \in(1,2), \\
\frac{x+4}{2}, & x \in[2,4] .
\end{array}\right.\right.
$$

It is clear that functions $f$ and $g$ of the present example satisfy all the conditions of the theorem with 1 is a fixed point. This satisfy E.A. property for sequences $1+\epsilon_{n}$ and $2+\epsilon_{n}$ where $\epsilon_{n} \rightarrow \infty$ whenever $n \rightarrow \infty$. To show weak semi compatibility and $f$-compatibility, a sequence $1+\epsilon_{n}$ is taken where $\epsilon_{n} \rightarrow \infty$ whenever $n \rightarrow \infty$. Since $\lim _{n \rightarrow \infty} f x_{n}=\lim _{n \rightarrow \infty} g x_{n}=2$ with $\lim _{n \rightarrow \infty} f g x_{n}=3=g(2)$ and $\lim _{n \rightarrow \infty} g f x_{n}=3 \neq f(2)$. Moreover, functions $f$ and $g$ satisfy $R$-weak commuting of type $A_{f}$. 
The next theorem is none else but just the generalization of above theorems in which both compatibility conditions are removed and extend one more commutativity condition, with assuming that functions satisfy E.A. property.

Theorem 3. Let $f$ and $g$ be two weak semi compatible self mappings of $G$-metric space $(X, G)$ satisfying following conditions:

(a) $f(X) \subseteq g(X)$;

(b) For every $x, y, z \in X$, holds

$$
\psi(G(g x, g y, g z)) \geq F(\psi(G(f x, f y, f z), \varphi(G(f x, f y, f z))) ;
$$

(c) $f$ and $g$ are either $R$-weak commuting of type $A_{f}$ or $A_{g}$;

(d) $f$ and $g$ satisfy E.A. property.

If $\varphi \in \Phi, \psi \in \Psi$ and $F \in \mathcal{C}_{\text {inv }}$ then $f$ and $g$ have a common fixed point in $X$.

Proof. Since $f$ and $g$ satisfy E.A. property then there exists a sequence $\left\{x_{n}\right\}$ in $X$ such that $\lim f x_{n}=\lim g x_{n}=t$ for some $t \in X$. Since $f(X) \subseteq g(X)$, then there exists a sequence $\left\{y_{n}\right\} \in X$ such that $\lim f x_{n}=\lim g y_{n}$ or $\lim f x_{n}=\lim g y_{n}=t$. Now by (b) we have

$$
\psi\left(G\left(g x_{n}, g y_{n}, g y_{n}\right)\right) \geq F\left(\psi\left(G\left(f x_{n}, f y_{n}, f y_{n}\right), \varphi\left(G\left(f x_{n}, f y_{n}, f y_{n}\right)\right)\right) .\right.
$$

Limiting $n \rightarrow \infty$ yields

$$
\psi(G(t, t, t)) \geq F\left(\psi\left(G\left(t, \lim f y_{n}, \lim f y_{n}\right), \varphi\left(G\left(t, \lim f y_{n}, \lim f y_{n}\right)\right)\right) .\right.
$$

This gives further

$$
\psi(0) \geq \psi\left(G\left(t, \lim f y_{n}, \lim f y_{n}\right)\right) .
$$

Since $\psi$ is a monotonic increasing function yields $\lim f y_{n}=t$. Thus

$$
\lim f x_{n}=t, \quad \lim g x_{n}=t, \quad \lim g y_{n}=t, \quad \lim f y_{n}=t .
$$

Case 1. If $f$ and $g$ are $R$-weak commuting of type $A_{g}$.

Since $f$ and $g$ are weak semi compatible mappings, this yields $\lim f g y_{n}=$ $g t$ or $\lim g f y_{n}=f t$. Let us first assume that $\lim f g y_{n}=g t$. It is clear by 31 that $\lim f g y_{n}=\lim f f x_{n}=g t$. Since $f$ and $g$ are $R$-weak commuting of type $A_{g}$, this yields

$$
d\left(g f x_{n}, f f x_{n}\right) \leq R d\left(f x_{n}, g x_{n}\right) .
$$

Limiting $n \rightarrow \infty$ yields

$$
d\left(\lim g f x_{n}, g t\right) \leq R d(t, t) .
$$

Thus $\lim g f x_{n}=g t$ or $\lim g g y_{n}=g t$. Now by (b)

$$
\psi\left(G\left(g g y_{n}, g t, g t\right)\right) \geq F\left(\psi\left(G\left(f g y_{n}, f t, f t\right), \varphi\left(G\left(f g y_{n}, f t, f t\right)\right)\right) .\right.
$$

Limiting $n \rightarrow \infty$ yields

$$
\psi(G(g t, g t, g t)) \geq F(\psi(G(g t, f t, f t), \varphi(G(g t, f t, f t))) .
$$


It implies $\psi(0) \geq \psi(G(g t, f t, f t))$ or $g t=f t$. Since $f$ and $g$ are $R$-weak commuting of type $A_{g}$ yields

$$
d(g f t, f f t) \leq R d(f t, g t)
$$

Since $g t=f t$, it gives $g f t=f f t$ or $g f t=f f t=f g t=g g t$. Now by $(\mathrm{b})$

$$
\psi(G(g f t, g t, g t)) \geq F(\psi(G(f f t, f t, f t), \varphi(G(f f t, f t, f t))) .
$$

This implies

$$
\begin{aligned}
\psi(G(f f t, g t, g t)) & \geq F(\psi(G(f f t, f t, f t), \varphi(G(f f t, f t, f t))) \\
& \geq \psi(G(f f t, g t, g t) .
\end{aligned}
$$

This gives $\psi(G(f f t, f t, f t)=0$ or $\varphi(G(f f t, f t, f t))=0$. This yields $f f t=$ $f t$ or $f f t=g f t=f t$. Thus, $f t$ is common fixed point of $f$ and $g$.

Next we suppose that $\lim g f y_{n}=f t$. Since $f$ and $g$ are $R$-weak commuting of type $A_{g}$, this yields

$$
d\left(g f y_{n}, f f y_{n}\right) \leq R d\left(f y_{n}, g y_{n}\right) .
$$

Limiting $n \rightarrow \infty$ gives

$$
d\left(f t, \lim f f y_{n}\right) \leq R d(t, t) \text { or } \lim f f y_{n}=f t .
$$

Since $f(X) \subseteq g(X)$, then there exists some $u \in X$ such that $f t=g u$. Therefore, $\lim g f y_{n}=\lim f f y_{n}=f t=g u$. Then by (b), we have

$$
\psi\left(G\left(g f y_{n}, g u, g u\right)\right) \geq F\left(\psi\left(G\left(f f y_{n}, f u, f u\right), \varphi\left(G\left(f f y_{n}, f u, f u\right)\right)\right) .\right.
$$

Limiting $n \rightarrow \infty$ yields

$$
\begin{aligned}
\psi(G(g u, g u, g u)) & \geq F(\psi(G(g u, f u, f u), \varphi(G(g u, f u, f u))) \\
& \geq \psi(G(g u, f u, f u))
\end{aligned}
$$

then

$$
\psi(0) \geq \psi(G(g u, f u, f u)) \text { or } g u=f u .
$$

Now, it can be easily shown that $f u$ is common fixed point of $f$ and $g$.

Case 2. If $f$ and $g$ are $R$-weak commuting of type $A_{f}$.

Since $f$ and $g$ are weak semi compatible mappings, this yields $\lim f g x_{n}=$ $g t$ or $\lim g f x_{n}=f t$. Let us first consider that $\lim f g x_{n}=g t$. Since $f$ and $g$ are $R$-weak commuting of type $A_{f}$, this gives

$$
d\left(f g x_{n}, g g x_{n}\right) \leq R d\left(f x_{n}, g x_{n}\right) .
$$

Limiting $n \rightarrow \infty$ yields

$$
d\left(g t, \lim g g x_{n}\right) \leq R d(t, t) \text { or } \lim g g x_{n}=g t .
$$

Now, by (b), we have

$$
\psi\left(G\left(g g x_{n}, g t, g t\right)\right) \geq F\left(\psi\left(G\left(f g x_{n}, f t, f t\right), \varphi\left(G\left(f g x_{n}, f t, f t\right)\right)\right) .\right.
$$

Limiting $n \rightarrow \infty$ yields

$$
\psi(G(g t, g t, g t)) \geq F(\psi(G(g t, f t, f t), \varphi(G(g t, f t, f t))) .
$$


This implies

$$
\psi(0) \geq F(\psi(G(g t, f t, f t), \varphi(G(g t, f t, f t))) \geq \psi(G(g t, f t, f t) .
$$

Since $\psi$ is a monotonic increasing, this yields $f t=g t$. Now it can be easily shown that $f t$ is common fixed point of $f$ and $g$.

Next we suppose that $\lim g f x_{n}=f t$. Since $\lim f x_{n}=\lim g y_{n}$ therefore $\lim g g y_{n}=f t$. Since $f(X) \subseteq g(X)$, then there exists a point $u$ in $X$ such that $f t=g u$. Since $f$ and $g$ are $R$-weak commuting of type $A_{f}$, yields

$$
d\left(f g y_{n}, g g y_{n}\right) \leq R d\left(f y_{n}, g y_{n}\right) .
$$

Limiting $n \rightarrow \infty$ yields

$$
d\left(\lim f g y_{n}, f t\right) \leq R d(t, t) .
$$

It yields $\lim f g y_{n}=f t$. Then we have $\lim g g y_{n}=\lim f g y_{n}=f t=g u$. Now, by (b), we have

$$
\psi\left(G\left(g g y_{n}, g u, g u\right)\right) \geq F\left(\psi\left(G\left(f g y_{n}, f u, f u\right), \varphi\left(G\left(f g y_{n}, f u, f u\right)\right)\right) .\right.
$$

Limiting $n \rightarrow \infty$ yields

$$
\psi(G(g u, g u, g u)) \geq F(\psi(G(g u, f u, f u), \varphi(G(g u, f u, f u))) .
$$

Hence, holds

$$
\psi(0) \geq F(\psi(G(g u, f u, f u), \varphi(G(g u, f u, f u))) \geq \psi(G(g u, f u, f u) .
$$

Since $\psi$ is monotonic increasing function, this yield $0 \geq G(g u, f u, f u)$ or $f u=g u$.

Now, it is very easy to show that $f u$ is common fixed point of $f$ and $g$.

Example 3. Let $(X, G)$ be a $G$-metric space where $G(x, y, z)$ be defined as $G(x, y, z)=|x-y|+|y-z|+|z-x|$, for all $x, y, z \in X$. Let $X=[2,6]$ and we define mapping $f, g: X \rightarrow X$ by

$$
f x=\left\{\begin{array}{cc}
4, & x \in[2,4], \\
2, & x \in(4,6],
\end{array} \quad g x=\left\{\begin{array}{cl}
x+2, & x \in(2,4), \\
x, & x \in[4,6] \text { or } x=2 .
\end{array}\right.\right.
$$

In the present example, the functions $f$ and $g$ satisfy all the given conditions of the theorem with 4 is fixed point. This satisfies E.A. property for sequence $2+\epsilon_{n}$ where $\epsilon_{n} \rightarrow \infty$ whenever $n \rightarrow \infty$. To show weak semi compatibility a sequence $2+\epsilon_{n}$ is taken where $\epsilon_{n} \rightarrow \infty$ whenever $n \rightarrow \infty$. Since $\lim _{n \rightarrow \infty} f x_{n}=\lim _{n \rightarrow \infty} g x_{n}=4$ with $\lim _{n \rightarrow \infty} f g x_{n}=2 \neq g(4)$ and $\lim _{n \rightarrow \infty} g f x_{n}=4=f(4)$. Moreover, functions $f$ and $g$ satisfy $R$-weak commuting of type $A_{f}$. 


\section{REFERENCES}

[1] M. Aamri and D. El. Moutawakil, Some new common fixed point theorems under strict contractive conditions, Journal of Mathmatical Analysis and applications, 270(1) (2002), 181-188.

[2] M. Abbas, N. Saleem, N. and M. De la Sen, Optimal coincidence point results in partially ordered non-Archimedean fuzzy metric spaces, Fixed point Theory and Applications, 1 (2016), 1-18.

[3] A. H. Ansari, Note on $\varphi$-contractive type mappings and related fixed point, The $2^{\text {nd }}$ Regional conference on Mathematics and Applications, Payame Noor University, $2014,377-380$.

[4] A. H. Ansari and A. Kaewcharoen, $C$-class functions and fixed point theorems for generalized $\alpha-\eta-\psi-\phi-F$-contraction type mappings in $\alpha-\eta$-complete metric space, Journal of Nonlinear Sciences and Applications, 9 (2016), 4177-4190.

[5] A. H. Ansari, M. A. Barakat and A. Ayadi, New approach for common fixed point theorems via $C$-class functions in $G_{p}$-metric spaces, Journal of Function Spaces, 2017 (2017), ArticleID 2624569, 9 pages.

[6] A. H. Ansari, H. Ayadi, M. Barakat and M. S. Khan, On coupled coincidence point results in partially ordered metric spaceeralized metric space, via generalized compatibility and $C$-class functions, Journal of Inequalities and Special Functions, 8(3) (2017), 125-138.

[7] A. H. Ansari, H. Ayadi, P. S. Kumari and I. Yildirim, New fixed point results via Cclass functions in b-rectangular metric spaces, Communication in mathematics and operations, 9 (2) (2018), 109-126.

[8] A. H. Ansari, N. Saleem, B. Fisher and M. S. Khan, C-class function on Khan type fixed point theorems in Genelaized metric space, Filomat, 31(11) (2017), 3483-3494.

[9] A. S. Ansari, W. Shatanawi, A. Kurdi and G. Maniu, Best proximity points in complete metric spaces with $(P)$-property via $C$-class functions, Journal of Mathematical Analysis, 7(6) (2016), 54-67.

[10] H. Ayadi, A. H. Ansari, B. Moeini, M. S. Noorani and H. Qawaqneh, Property Q on $G$-metric spaces via C-class function, International Journal of Mathematics and Computer Science, 14(3) (2019), 675-692.

[11] B. C. Dhage, Generalized metric space and mapping with fixed point, Bulletin of the Calcutta Mathematical Society, 84(4) (1992), 329-336.

[12] S. Gahler, 2-metrische Raume and ihre topologische structure, Mathematische Nachrichten, 26 (1963), 115-148.

[13] Z. Mustafa, Common Fixed Points of Weakly Compatible Mappings in G-Metric spaces, Applied Mathematical Sciences, 6 (92) (2012), 4589-4600.

[14] Z. Mustafa and B. Sims, Some remarks concerning D-metric spaces, In: J. GarciaFalset et al. (Eds), Proceeding of the International conferences on fixed point theory and applications, July 13-19, Yokohama Publishers, Velencia, Spain, Yokohama Publishers, Yokohama 2004, pp. 189-198. 
[15] Z. Mustafa and B. Sims, A new approach to generalized metric spaces, Journal of Nonlinear and Convex Analysis, 7(2) (2006), 289-297.

[16] Z. Mustafa and B. Sims, Fixed point theorems for contractive mappings in Gmetric spaces, Fixed Point Theory and Applications, 2009 (2009), ArticleID 917175, 10 pages.

[17] Z. Mustafa, F. Awahdeh and W. Shatanawi, Fixed point theorem for expansive mappings in G-metric space, International Journal of Contemporary Mathematical Sciences, 5(50) (2010), 2463-2472.

[18] Z. Mustafa, H. Aydi and E. Karapinar, On common fixed points in G-metric spaces using (EA) property, Computers \& Mathematics with Applications, 6(6) (2012), 1944-1956.

[19] Z. Mustafa, H. Aydi and E. Karapinar, Generalized Meir-Keeler type contractions on G-metric spaces, Applied Mathematics and Computation, 219 (2013), 10441-10447.

[20] Z. Mustafa, H. Obiedat and F. Awahdeh, Some fixed point theorem for mapping on complete G-metric space, Fixed Point Theory and Applications, 2008 (2008), ArticleID 189870, 12 pages.

[21] Z. Mustafa, W. Shatanwi and M. Bataineh, Fixed point theorem on uncomplete Gmetric spaces, Journal of Mathematics and Statistics, 4(4) (2008), 196-201.

[22] H. K. Pathak and M. S. Khan, A comparison of various types of compatible maps and common fixed points, Indian journal of Pure and Applied Mathematics, 28 (4) (1997), 477-485.

[23] H. K. Pathak, Y. J. Cho and S. M. Kang, Remarks of R-weakly commuting mappings and common fixed point theorems, Bulletin of the Korean Mathematical Society, 34 (1997), 247-257.

[24] N. Saleem, A. H. Ansari and M. K. Jain, Some fixed point theorems of inverse C-class function under weak semi compatibility, Journal of Fixed Point Theory, 2018 (2018), ArticleID 9, 23 pages.

[25] A. S. Saluja, M. K. Jain and P. K. Jhade, Weak semi compatibility and fixed point theorems, Bulletin of International Mathematical virtual Institute, 2(2) (2012), 205-217.

[26] M. R. Singh and Y. M. Singh, On various types of compatible maps and common fixed point theorem for non-continuous maps, Hacettepe Journal of Mathematics and Statistics, 40(4) (2011), 503-513. 


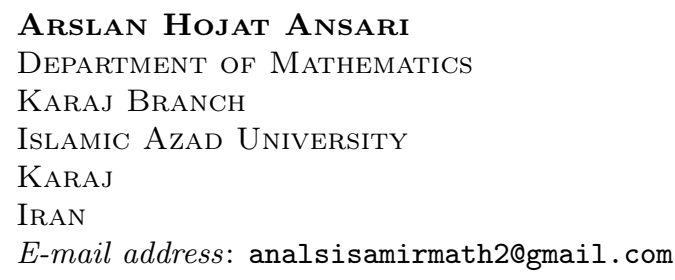

\section{Mukesh Kumar Jain}

JaWAHAR NaVodaya Vidyalaya

UDALGURI ASSAM

INDIA

E-mail address: mukesh.jain2007@rediffmail.com

\section{NaEem Saleem}

Nonlinear Analysis Research Group

Ton Duc Thang University

Ho Chi Minh City

VIETNAM

Department of Mathematics and Statistics

Ton Duc Thang University

Ho Chi Minh City

VIETNAM

E-mail address: naeem.saleem@tdtu.edu.vn

naeem.saleem2@gmail.com 